\title{
Antoni van Leeuwenhoek
}

Homme d'affaires néerlandais, physicien dilettante très créatif, Antoni van Leeuwenhoek est connu pour ses améliorations du microscope et est considéré comme l'un des précurseurs de la microbiologie. II est en effet le premier à avoir fait des observations étonnantes avec un microscope qu'il s'est luimême construit, et qui lui a permis de découvrir les bactéries, les cellules du sang et la circulation dans les capillaires, ainsi que les organismes eucaryotes, entre autres. Mieux encore: après avoir longtemps été correspondant de la Royal Society de Londres, ce savant si peu ordinaire, qui n'a reçu aucune formation universitaire et ne parle que son néerlandais natal, en devient l'un des membres les plus actifs.

Riad HAIDAR, haidar@onera.fr

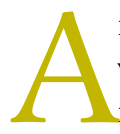

ntoni (également orthographié Anthonie ou Antonie, voire Theunis ou Thonis) Philips van Leeuwenhoek naît à Delft, au sein de la jeune république des Provinces-Unies (ancêtre des actuels Pays-Bas), le 24 octobre 1632, pendant ce que l'on appelle le siècle d'or néerlandais.

Son père, Philips Antonisz van Leeuwenhoek, est un fabricant de paniers et sa mère, Margaretha BeL van den Berch, est issue d'une famille de brasseurs aisés. Antoni a quatre sœurs plus âgées (Margriet, Geertruyt, Neeltje et Catharina). Suite à la disparition de son père en 1637, sa mère épouse Jacob Jansz Molijn, un peintre: la fratrie reste ainsi unie, jusqu'au décès de Jacob en 1642. Antoni est envoyé à l'école de Warmond pendant une courte période, puis il est confiéà son oncle, un avocat qui exerce à Benthuizen, un petit village à quelques kilomètres de Delft. À l'âge de 16 ans, il entre au service de William Davidson, un marchand de draps établi à Amsterdam, au titre d'apprenti-comptable.

À l'été 1654, il épouse Barbara de Mey; de cette union naîtront 5 enfants. Puis il retourne définitivement à Delft, et s'établit à son tour comme marchand de draps et de produits de mercerie. Bien que l'on n'en ait pas de traces, il est probable que son commerce soit prospère et qu'il

\section{Le saviez-vous?}

Version frugale du microscope de van Leeuwenhoek, le foldscope est un microscope inventé en 2012 par Manu Prakash, professeur à l'université Stanford. Il s'agit d'un microscope en papier plié (folded paper, en anglais) vendu à un prix minime (moins d'un dollar américain) et permettant de grossir jusqu'à plus de 2000 fois.

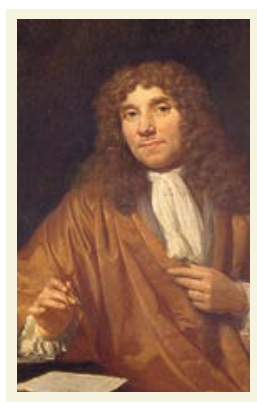
PRINCIPALES DATES 24 octobre 1632 - Naissance à Delft (Pays-Bas) 1673 Publication du premier article 1676 Découverte des micro-organismes 1680 Élection à la Royal Society 1689 Découverte de la circulation dans les capillaires 26 août 1723 - Décès à Delft (Pays-Bas)

Portrait d'Antoni van Leeuwenhoek

acquière un certain statut social. Il fréquente Jan Vermeer, le grand peintre baroque également originaire et établi à Delft, dont il sera l'exécuteur testamentaire en 1675. En 1660, il devient chambellan de l'échevin à L' hôtel de ville de Delft; il s'agit d'un poste lucratif qu'il occupera pendant près de 40 ans. Sa femme Barbara décède en 1666, et il épouse Cornelia Swalmius en 1671 en secondes noces. En 1669, Antoni est nommé arpenteur par la Cour de Hollande, et occupe pendant quelques temps la responsabilité de wynroeyer municipal (littéralement, jaugeur des barils de vin), en charge du contrôle des importations de vin à Delft.

\section{Techniques et découvertes}

L'intérêt d'Antoni van Leeuwenhoek pour la microscopie est d'abord motivé par son métier de drapier. Inspiré par le magnifique et très populaire livre illustré de Robert Hooke, Micrographia, paru en 1665, il souhaite en effet vérifier la qualité de la toile qu'il utilise pour ses draps. Il semble que ce soit vers 1668 qu'il se forme à l'art délicat du polissage du verre pour construire ses propres instruments grossissants. Très vite, il développe une technique nouvelle (et qu'il gardera jalousement secrète) pour la fabrication

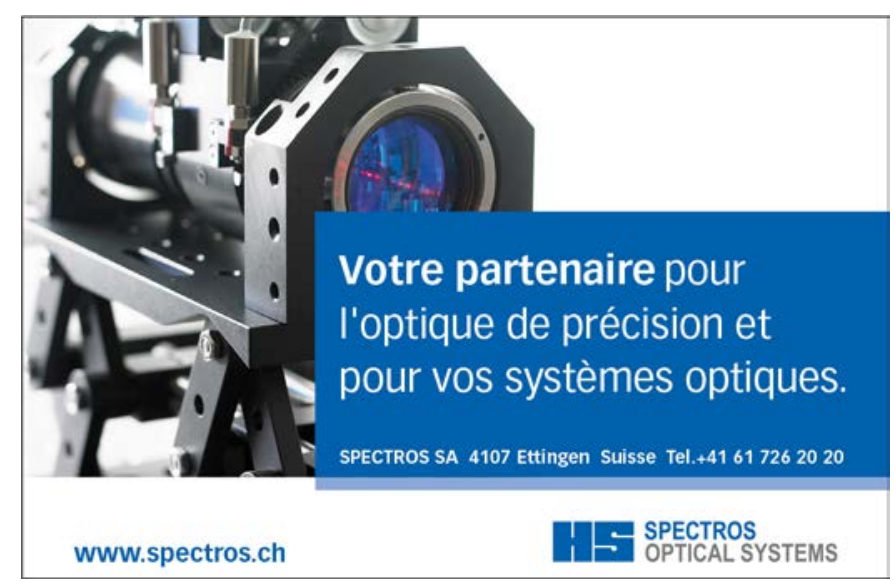




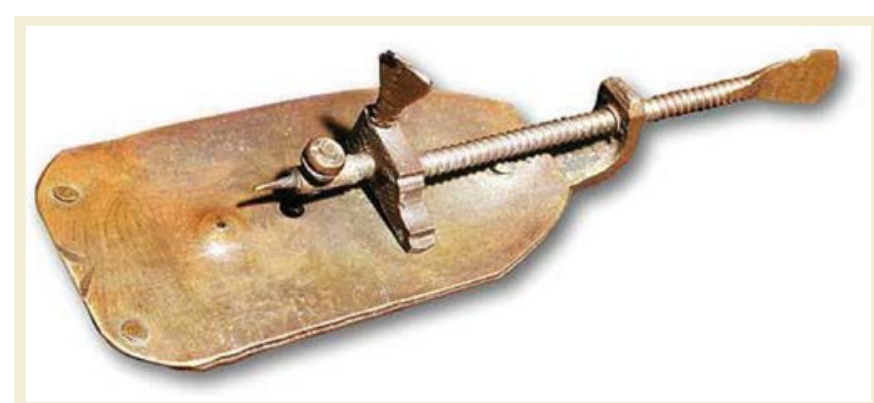

Microscope de van Leeuwenhoek, d'une taille d'environ $5 \mathrm{~cm}$. La lentille est placée dans le trou et l'objet, piqué sur la pointe en métal, est déplacé à l'aide des 3 vis devant la lentille. L'ensemble est tenu très près de l'œil, face à la lumière, et permet d'observer des objets de quelques microns.

de petites sphères de verre de haute qualité, qu'il utilise comme lentilles optiques de forte puissance. Il en construit ainsi plusieurs centaines, et met au point plus de $25 \mathrm{mi}$ croscopes à lentilles uniques, dont 9 nous sont parvenus presque intacts. Ses instruments sont composés d'un châssis de cuivre ou d'argent, et présentent des performances remarquables (grossissement de $275 \times$ à probablement $500 \times$ ), exceptionnelles pour l'époque, et qui ne seront égalées que près d'un siècle plus tard.

\section{Premières observations au microscope}

Dans les années 70, Antoni fait ses premières observations de l'univers microscopique: il étudie tout ce qui passe, les poux, les abeilles, le sang... Il n'a pas de formation technique, et pas véritablement de méthode, simplement une immense curiosité scientifique et un indéniable talent expérimental. Il découvre ainsi un monde littéralement inconnu, qu'il dévoile à l'un des amis, Reinier de Graaf, médecin réputé. De Graaf, immédiatement conquis, décide de faire connaître les travaux de van Leeuwenhoek, et adresse un courrier à l'éditeur des Philosophical Transactions de la Royal Society de Londres. À son tour, celui-ci perçoit la portée et l'originalité de ces observations, et convainc Van Leeuwenhoek de publier un article dans son journal. Nous sommes en 1673: Antoni vient, en parfait dilettante, de faire une entrée remarquée dans l'univers des savants. L'article de 1673 est le premier d'une longue série de lettres - toutes écrites dans un néerlandais familier, le seul que connaît van Leeuwenhoek, et décrivant un travail qu'il s'obstine à faire en solitaire, refusant toute assistance. Elles sont ensuite traduites en latin ou en anglais par l'éditeur des Philosophical Transactions, Henry Oldenburg, qui a dû apprendre le néerlandais pour l'occasion...

L'histoire est savoureuse: van Leeuwenhoek cherche à observer les "petites aiguilles» qui pourraient expliquer l'effet du poivre sur la langue, et découvre accidentellement ces petits êtres connus aujourd'hui sous le nom de protozoaires.
En 1676, toutefois, la crédibilité de van Leeuwenhoek est ébranlée: il vient de communiquer ses premières observations d'organismes vivants microscopiques ${ }^{1}$, qui étaient alors inconnus - une découverte qui, si elle se confirme, contredit toutes les théories biologiques alors en vigueur. Sans surprise, elle est puissamment contestée, voire tournée en ridicule par l'assemblée des savants londoniens. L'accusation est grave, et renforcée par le fait que nul ne peut vérifier les dires de van Leeuwenhoek, puisque lui seul dispose de microscopes assez puissants pour observer ce qu'il prétend avoir vu. Mais van Leeuwenhoek s'obstine et la Royal Society se décide à missionner un groupe d'experts à Delft, dont un certain William Croone, médecin éminent. En 1677, guidés par van Leeuwenhoek qui leur ouvre les portes de son laboratoire, ces experts impartiaux se rendent à l'évidence et confirment l'existence des micro-organismes vivants.

\section{Reconnaissance scientifique}

Antoni van Leeuwenhoek est élu au rang de membre de la Royal Society en février 1680, sous le parrainage influent de William Croone qui, on s'en souvient, faisait partie des experts missionnés à Delft. Sa notoriété est désormais considérable, et de nombreuses personnalités lui rendent visite pour voir les créatures minuscules: Leibniz, Guillaume III d'Orange-Nassau et son épouse, le maire d'Amsterdam Johan Huydecoper, ou encore le Tsar Pierre le Grand de Russie. De nombreux admirateurs, anonymes ou non, lui envoient des objets à observer... Notons qu'en 1689, il démontre la circulation du sang dans les capillaires. Travailleur infatigable, il continue ainsi et accumule un nombre considérable d'observations sur les micro-organismes jusqu'à sa mort. Il décède le 26 août 1723, à presque 91 ans, et est inhumé dans la Vieille Église de Delft.

\section{Invention du microscope}

L'origine du microscope est controversée. Certains la situent à la fin du $16^{\mathrm{e}}$ siècle, et l'attribuent à Zacharias Janssen, un fabricant hollandais de lentilles. D'autres défendent la paternité de Galilée, en Italie, qui a eu l'idée de combiner une lentille convexe et une lentille concave en 1609 pour obtenir un pouvoir grossissant. Quoi qu'il en soit, l'un des utilisateurs les plus célèbres est l'anglais Robert Hooke, auteur de Micrographia en 1665. Enfin, les historiens s'accordent à attribuer à Antoni van Leeuwenhoek d'avoir porté le microscope à la connaissance des biologistes, grâce à ses observations qui ont bouleversé leur perception du vivant.

\section{RÉFÉRENCES}

[1] Maria Rooseboom, Leeuwenhoek, the Man: A Son of His Nation and His Time, Bulletin of the British Society for the History of Science (1950).

[2] Jean Rostand, La Genèse de la vie. Histoire des idées sur la génération spontanée (1943). 